\title{
Expanding Verrucous Plaque on the Face
}

\author{
Alyssa Miceli, DO; Ann Church, MD; Karthik Krishnamurthy, DO
}

\begin{abstract}
Eligible for 1 MOC SA Credit From the ABD
This Photo Challenge in our print edition is eligible for 1 self-assessment credit for Maintenance of Certification from the American Board of Dermatology (ABD). After completing this activity, diplomates can visit the ABD website (http://www.abderm.org) to self-report the credits under
\end{abstract} the activity title "Cutis Photo Challenge." You may report the credit after each activity is completed or after accumulating multiple credits.
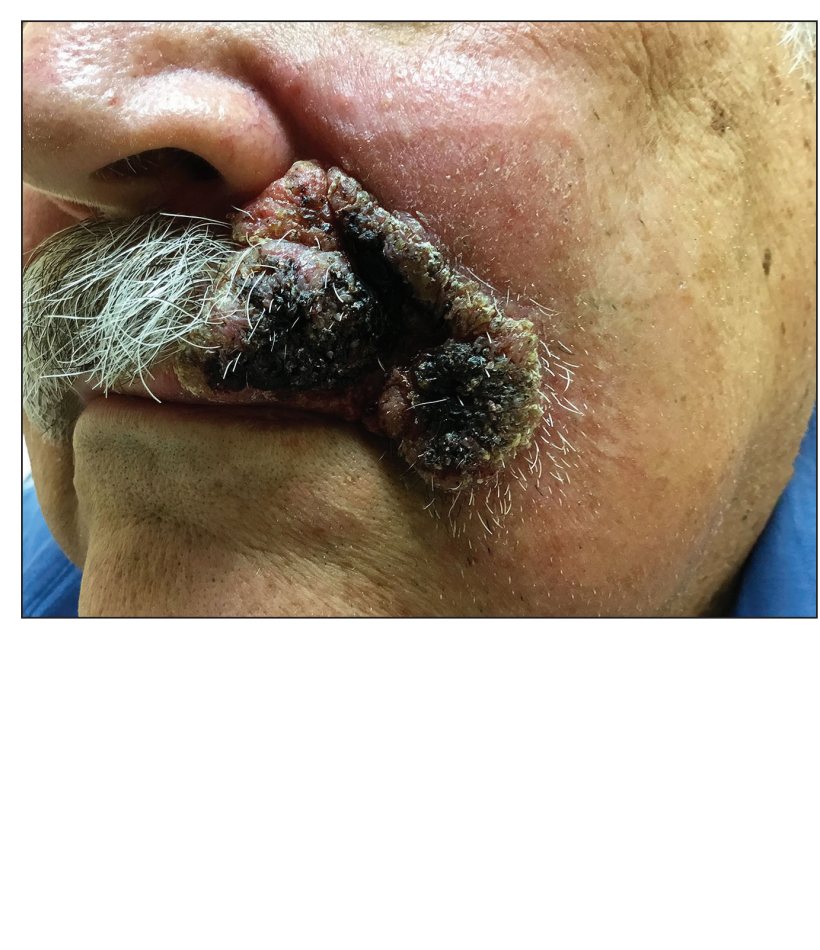

A 69-year-old man presented with a slowly expanding, verrucous plaque on the left side of the upper cutaneous lip of 4 months' duration. The lesion reportedly began as an abscess and had undergone incision and drainage followed by multiple courses of oral antibiotics that were unsuccessful prior to presentation to our clinic. The patient's hobbies included gardening near his summer home in the mountains of western North Carolina, where he resided when the lesion appeared. Physical examination revealed an approximately $6 \times 4-\mathrm{cm}$ verrucous plaque with central ulceration on the left side of the upper cutaneous and vermilion lip extending to the nasolabial fold. A review of systems was negative for any systemic symptoms. Routine laboratory tests and computed tomography of the head and neck were normal.

\section{WHAT'S YOUR DIAGNOSIS?}
a. blastomycosis
b. chromoblastomycosis
c. coccidioidomycosis
d. leishmaniasis
e. pemphigus vegetans

Drs. Miceli and Krishnamurthy are from Orange Park Medical Center, Florida. Dr. Church is from Aurora Diagnostics Bernhardt Laboratories, Jacksonville, Florida.

The authors report no conflict of interest.

Correspondence: Alyssa Miceli, DO, Orange Park Medical Center, 906 Park Ave, Orange Park, FL 32073 (alyssa.miceli@gmail.com). doi:10.12788/cutis.0154 


\section{THE DIAGNOSIS: Blastomycosis}

$\mid$ istopathologic examination of 3 punch biopsies from the left side of the upper lip showed pseudoepitheliomatous hyperplasia with intraepidermal microabscesses and dermal suppurative granulomatous inflammation (Figure 1A). Stains were negative for periodic acid-Schiff, herpes simplex virus, and varicellazoster virus. Direct and indirect immunofluorescence for skin autoantibodies were negative. Two separate tissue culture specimens showed no bacterial, fungal, or mycobacterial growth. Leishmania polymerase chain reaction and DNA sequencing were negative. An additional punch biopsy revealed yeast forms with broad-based budding and refractile walls (Figures $1 \mathrm{~B}$ and $1 \mathrm{C}$ ) that were highlighted with Grocott-Gomori methenamine-silver stain of the tissue (Figure 2). Chest radiography demonstrated no pulmonary involvement. In collaboration with an infectious disease specialist, the patient was started on itraconazole $200 \mathrm{mg}$ twice daily for a total of 6 months.

Blastomycosis is a fungal infection caused by Blastomyces dermatitidis, a thermally dimorphic fungus endemic in the soils of the Ohio and Mississippi River valleys and southeastern United States. ${ }^{1}$ It most commonly manifests as a pulmonary infection following inhalation of spores that are transformed into thick-walled yeasts capable of evading the host's immune system. Unlike other deep fungal infections, blastomycosis occurs in both immunocompetent and immunocompromised hosts. Extrapulmonary disease after hematogenous dissemination from the lungs occurs in approximately $25 \%$ to $30 \%$ of patients, with the skin as the most common site of dissemination. ${ }^{2}$ Clinically, cutaneous blastomycosis typically starts as papules that evolve into crusted vegetative plaques, often with central clearing or ulceration. Primary cutaneous blastomycosis is rare and occurs due to direct inoculation after trauma to the skin via an infected animal bite, direct inoculation in laboratory settings, or due to injury during outdoor activities involving contact with soil. ${ }^{3}$ Given our patient's horticultural hobbies, lack of pulmonary symptoms, and negative radiologic examination, primary cutaneous blastomycosis infection due to direct inoculation from contaminated soil was a possibility; however, definite confirmation was difficult, as the primary pulmonary infection of blastomycosis can be asymptomatic and therefore often goes undetected.

Cutaneous blastomycosis can be mistaken for pemphigus vegetans, leishmaniasis, herpes vegetans, bacterial pyoderma, and other deep fungal infections that also display pseudoepitheliomatous hyperplasia with pyogranulomatous inflammation on histopathology. Direct visualization of the characteristic yeast forms in a histologic specimen or the growth of fungus in culture is essential for a definitive diagnosis. The yeasts are 8 to $15 \mu \mathrm{m}$ in diameter with thick, double-contoured walls
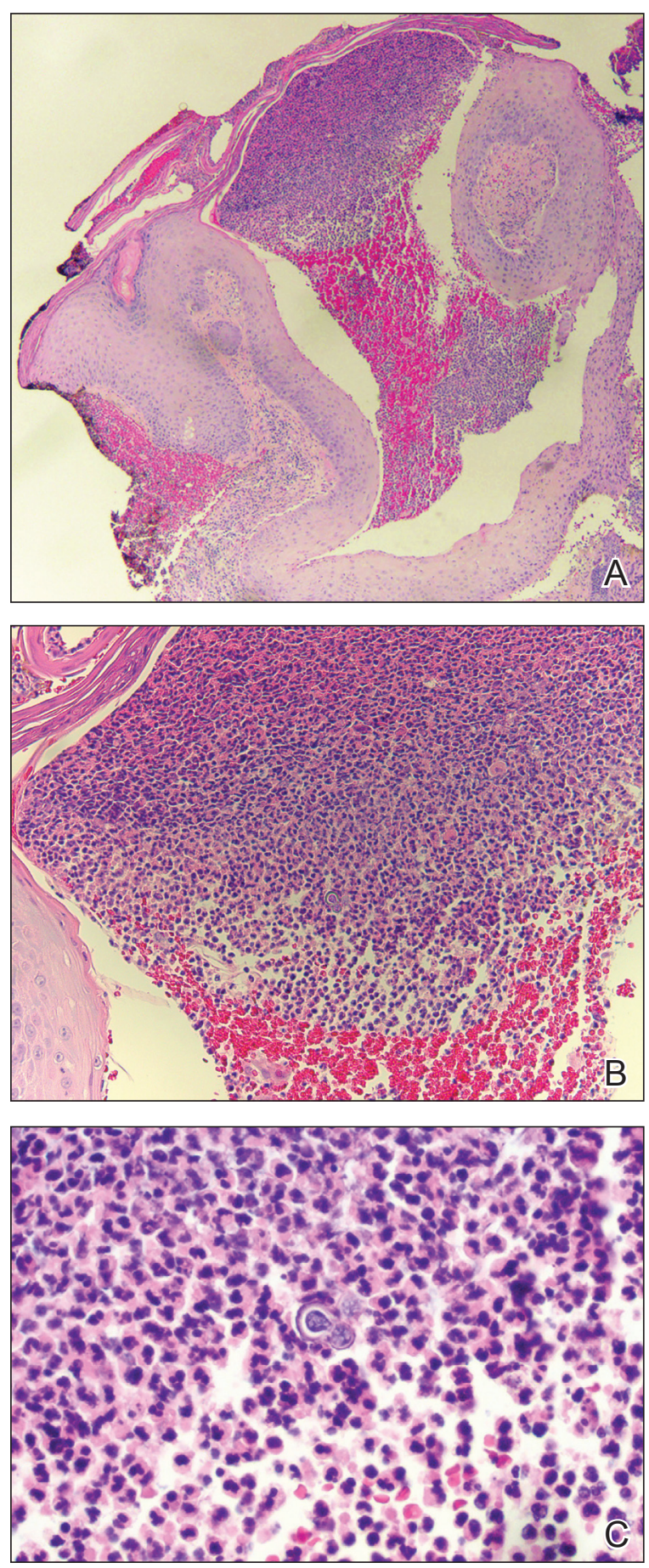

FIGURE 1. Blastomycosis. A, Histopathology revealed pseudoepitheliomatous hyperplasia with intraepidermal microabscesses and dermal suppurative granulomatous inflammation ( $\mathrm{H} \& \mathrm{E}$, original magnification $\times 20$ ). $\mathrm{B}$ and $\mathrm{C}$, Yeast forms with broad-based budding and refractile walls within microabscesses $(H \& E$, original magnifications $\times 200$ and $\times 400)$. 


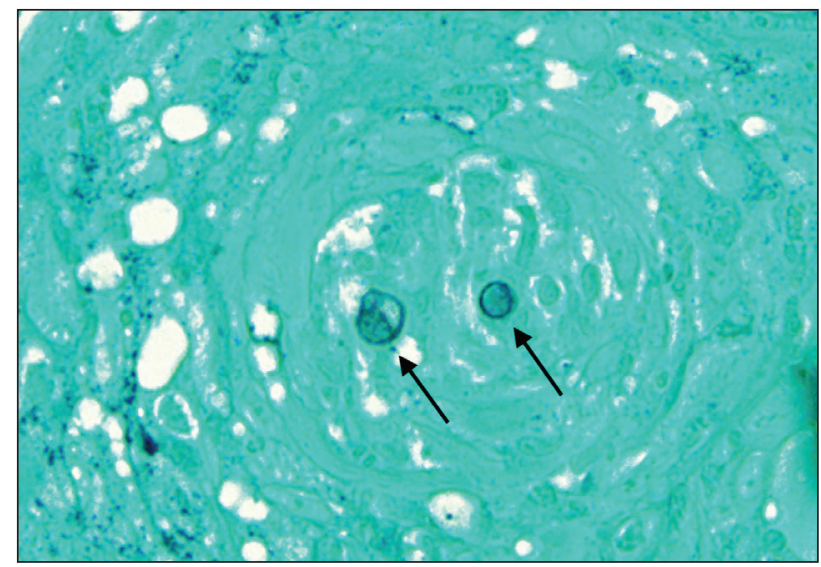

FIGURE 2. Grocott-Gomori methenamine-silver stain highlighted nonbudding yeast forms (arrows)(original magnification $\times 400$ ).

and characteristically display broad-based budding. ${ }^{4}$ This budding pattern aids in differentiating blastomycosis from other entities with a similar histopathologic appearance. Chromoblastomycosis would show brown, thick-walled fungal cells inside giant cells, while coccidioidomycosis displays large spherules containing endospores, and leishmaniasis demonstrates amastigotes (small oval organisms with a bar-shaped kinetoplast) highlighted with Giemsa staining. Pemphigus vegetans would show intercellular deposition of IgG on direct immunofluorescence. Blastomyces dermatitidis can be difficult to visualize with routine hematoxylin and eosin stains, and it is important to note that a negative result does not exclude the possibility of blastomycosis, as demonstrated in our case. ${ }^{4}$ Special stains including Grocott-Gomori methenaminesilver and periodic acid-Schiff can aid in examining tissue for the presence of fungal elements, which typically can be found within histiocytes or abscesses in the dermis. Culture is the most sensitive method for detecting and diagnosing blastomycosis. Growth typically is detected in 5 to 10 days but can take up to 30 days if few organisms are present in the specimen. ${ }^{1}$

Although spontaneous remission can occur, it is recommended that all patients with cutaneous blastomycosis be treated to avoid dissemination and recurrence. Itraconazole currently is the treatment of choice. ${ }^{5}$ Doses typically are 200 to $400 \mathrm{mg} / \mathrm{d}$ for 8 to 12 months. ${ }^{6}$ Itraconazole-related side effects experienced by our patient during his 6-month treatment course included leg edema, 20-lb weight gain, gastrointestinal upset, blurred vision, and a transient increase in blood pressure, all resolving once the medication was discontinued. Complete resolution of the lesion was noted at the completion of the treatment course. At a 6-month posttreatment follow-up, residual scarring and alopecia were noted in parts of the previously affected areas of the upper cutaneous lip and nasolabial fold.

\section{REFERENCES}

1. Saccente M, Woods GL. Clinical and laboratory update on blastomycosis. Clin Microbiol Rev. 2010;23:367-831.

2. Chapman SW, Lin AC, Hendricks KA, et al. Endemic blastomycosis in Mississippi: epidemiological and clinical studies. Semin Respir Infect. 1997;12:219-228.

3. Gray NA, Baddour LM. Cutaneous inoculation blastomycosis. Clin Infect Dis. 2002;34:E44-E49.

4. Patel AJ, Gattuso P, Reddy VB. Diagnosis of blastomycosis in surgical pathology and cytopathology: correlation with microbiologic culture. Am J Surg Pathol. 2010;34:256-261.

5. Chapman SW, Dismukes WE, Proia LA, et al. Clinical practice guidelines for the management of blastomycosis: 2008 update by the Infectious Diseases Society of America. Clin Infect Dis. 2008;46:1801-1812.

6. Lomaestro, BM, Piatek MA. Update on drug interactions with azole antifungal agents. Ann Pharmacother. 1998;32:915-928. 